\title{
Study on Laser Communication: Features, Application, Advantages
}

\author{
Prof. Atul A. Padghan \\ Principal, Electronics and Telecommunication \\ Department, Dr.R.N.Lahoti Polytechnic, \\ Sultanpur, Uttar Pradesh
}

\author{
Prof. Ankit P. Jaiswal \\ HOD, Mechanical Engg Department, Dr.R.N.Lahoti \\ Polytechnic, Sultanpur, Uttar Pradesh
}

\section{ABSTRACT}

Laser communications offer a viable alternative to RF communications for intersatellite links and other applications where high-performance links are necessary. High data rate, small antenna size, narrow beam divergence, and a narrow field of view are characteristics of laser communication that offer a number of potential advantages for system design. The high data rate and large information throughput available with laser communications are many times greater than in radio frequency (RF) systems. The small antenna size requires only a small increase in the weight and volume of host vehicle. In addition, this feature substantially reduces blockage of fields of view of the most desirable areas on satellites. The smaller antennas, with diameters typically less than $30 \mathrm{~cm}$, create less momentum disturbance to any sensitive satellite sensors. The narrow beam divergence of affords interference-free and secure operation.

\section{INTRODUCTION}

Lasers have been considered for space communications since their realization in 1960. However, it was soon recognized that, although the laser had potential for the transfer of data at extremely high rates, specific advancements were needed in component performance and systems engineering, particularly for space-qualified hardware. Advances in system architecture, data formatting, and component technology over the past three decades have made laser communications in space not only a viable but also an attractive approach to intersatellite link applications. The high data rate and large information throughput available with laser communications are many times greater than in radio frequency (RF) systems. The small antenna size requires only a small increase in the weight and volume of host vehicle. In addition, this feature substantially reduces blockage of fields of view of the most desirable areas on satellites. The smaller antennas, with diameters typically less than $30 \mathrm{~cm}$, create less momentum disturbance to any sensitive satellite sensors. Fewer onboard consumables are required over the long lifetime because there are fewer disturbances to the satellite compared with larger and heavier RF systems. The narrow beam divergence of affords interference-free and secure operation.

\subsection{FEATURES OF LASER COMMUNICATIONS SYSTEM}

A block diagram of typical terminal is illustrated in Fig 1. Information, typically in the form of digital data, is input to data electronics that modulates the transmitting laser source. Direct or indirect modulation techniques may be employed depending on the type of laser employed. The source output passes through an optical system into the channel. The optical system typically includes transfer, beam shaping, and telescope optics. The receiver beam comes in through the optical system and is passed along to detectors and signal processing electronics. There are also terminal control electronics that must control the gimbals and other steering mechanisms, 
and servos, to keep the acquisition and tracking system operating in the designed modes of operation.

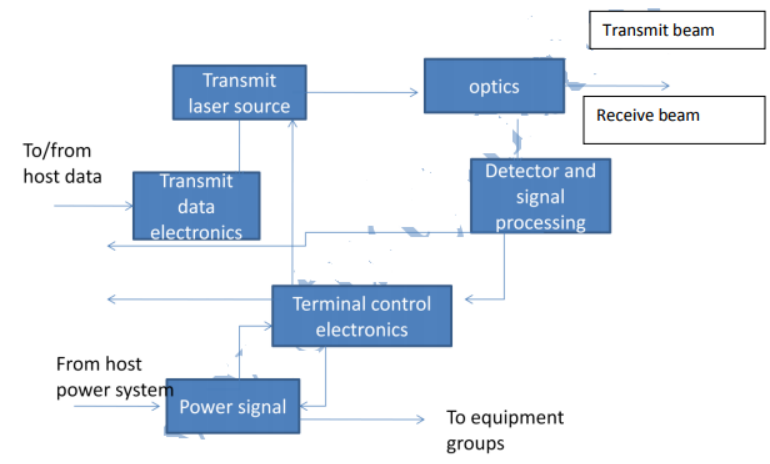

Figure 1.1 A block diagram of a typical laser communication terminal.

When the receiver is detecting signals, it is actually making decisions as to the nature of the signal (when digital signal are being sent it distinguishes between ones and zeros). Fig 3. shows the probability of detection vs. measured photocurrent in a decision time. There are two distributions: one when a signal is present (including the amount of photocurrent due to background and dark current in the detector), and one when there is no signal present (including only the non signal current sources). A threshold must be set that maximizes the success rate and minimizes the error rate. One can see that different types of errors will occur. Even when there is no signal present, the fluctuation of the non signal sources will periodically cause the threshold to be exceeded. This is the error of stating that a signal is present when there is no signal present. The signal distribution may also fall on the other side of the threshold, so errors stating that no signal is present will occur even when a signal is present. For laser communication systems in general, one wants to equalize these two error types. In the acquisition mode, however, no attempt is made to equalize these errors since this would increase acquisition time.

\subsection{OPERATION}

Free space laser communications systems are wireless connections through the atmosphere. They work similar to fibre optic cable systems except the beam is transmitted through open space. The carrier used for the transmission of this signal is generated by either a high power LED or a laser diode. The laser systems operate in the near infrared region of the spectrum. The laser light across the link is at a wavelength of between $780-920 \mathrm{~nm}$. Two parallel beams are used, one for transmission and one for reception.

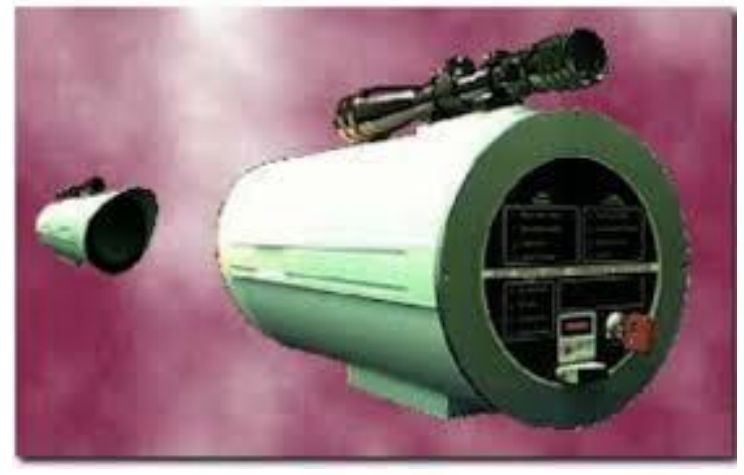

Fig 1.2 MAGNUM 45 High-Speed LaserCommunication Systems (Source: LSA Photonics)

\subsection{LINK PARAMETERS}

The link parameters include the type of laser, wavelength, type of link, and the required signal criterion. Today the lasers typically used in free space laser communications are the semiconductor laser diodes, solid state lasers, or fibre amplifier lasers. Laser sources are described as operating in either in single or multiple longitudinal modes. In the single longitudinal mode operation the laser emits radiation at a single frequency, while in the multiple longitudinal mode, multiple frequencies are emitted. Semiconductor lasers have been in development for three decades and have only recently (within the past 7 years) demonstrated the levels of performance needed for the reliable operation as direct sources typically operating in the $800-900 \mathrm{~nm}$ range(gallium arsenide/gallium aluminium arsenide)their inherently high efficiency (50\%)and small size made this technology attractive. The key issues have been the life times, asymmetric beam shapes, output power. Solid state lasers have offered higher power levels and the ability to operate in high peak power modes for the acquisition. When laser diodes are used to optically pump the lasing media graceful degradation and higher overall reliability is achieved. A variety of materials have been proposed for laser transmitters: neodyminium doped yttrium aluminium garnet $(\mathrm{Nd}$ : YAG) is the most widely used. Operating at $1064 \mathrm{~nm}$, these lasers require an external modulator leading to a slight increase in the complexity and reliability. With the rapid development of terrestrial fibre communications, a wide array of components is available for the potential applications in space. These include detectors, lasers, multiplexers, amplifiers, optical pre amplifiers etc. 


\subsection{TRANSMITTER PARAMETERS}

The transmitter parameter consists of certain key laser characteristics, losses incurred in the transmitter optical path, transmit antennae gain, and transmit pointing losses. The key laser characteristics include peak and average optical power, pulse rate and pulse width. In a pulsed configuration the peak laser power and duty cycle are specified, whereas in continuous wave application, the average power is specified. Transmit optical path loss is made up of optical transmission losses and the loss due to the wave front quality of the transmitting optics. The wave front error loss is analogous to the surface roughness loss associated with the RF antennas. The optic transmit antenna gain is analogous to the antenna gain in the RF systems and describes the on axis gain relative to an isotropic radiator with the distribution of the transmitted laser radiation defining the transmit antenna gain. The laser sources suitable for the free space communications tend to exhibit a Gaussian intensity distribution in the main lobe. The reduction in the far field signal strength due to the transmitter pointing is the transmitter pointing losses. The pointing error is composed of bias (slowly varying) and random (rapidly varying) components.

\subsection{CHANNEL PARAMETERS}

The channel parameters for an optical intersatellite link(ISL) consist of range and associated loss ,background spectral radiance and spectral irradiance. The range loss is directly proportional to the square of wavelength and inversely proportional to the square of the separation between the platform in metres.

\subsection{RECEIVER PARAMETERS}

The receiver parameters are the receiver antenna gain, the receive optical path loss, the optical filter bandwidth and the receiver field of view. The receiver antenna gain is proportional to the square of effective receiver diameter in metres and inversely proportional to the square of the wavelength. The receiver optical path loss is simply the optical transmission loss for systems employing the direct detection techniques. However for the lasers employing the coherent optical detection there is an additional loss due to the wave front error. The preservation of the wave front quality is essential for the optimal mixing of the received signal and the local oscillator fields on the detector surface. The optical filter bandwidth specifies the spectral width of the narrow band pass filter employed in optical inter satellite links. Optical filters reduce the amount of unwanted background entering the system. The optical width of the filter must be compatible with the spectral width of the laser source. The minimum width will be determined by the acceptable transmission level of the filter. The final optical parameter is the angular field of view (FOV), in radians which limits the background power of an extended source incident on the detector. To maximize the rejection, the FOV should be as small as possible. For small angles the power incident on the detector is proportional to FOV square. The minimum FOV is limited by optical design constraints and the receiver pointing capability.

\subsection{DETECTOR PARAMETERS}

The detector parameters are the type of detector, gain of detector, quantum efficiency, heterodyne mixing efficiency, noise due to the detector, noise due to the following pre amplifier and angular sensitivity. For optical ISL systems based on semiconductor laser diodes or Nd:YAG lasers the detector of choice is a $\mathrm{p}$ type intrinsic $n$ type (PIN) or an avalanche photodiode(APD) APIN photo diode can be operated in the photovoltaic or photoconductive mode and has no internal gain mechanism. An APD is always operated in the photo conductive mode and has an internal gain mechanism, by virtue of avalanche multiplication. The quantum efficiency of the detector is the efficiency with which the detector converts the incident photons to electrons. The mean output current for both the PIN and APD is proportional to the quantum efficiency.

\section{APPLICATIONS}

Depending on the climatic zone where the free space laser communications systems are used, they can span distances up to $15 \mathrm{~km}$ at low bitrates or provide bitrates up to $622 \mathrm{Mbps}$ at shorter distances. The systems are protocol transparent allowing transmission of digital computer data (LAN interconnect), video, and voice over IP, multiplexed data, or ATM. They are suitable for temporary connectivity needs such as at conventions, sporting events, corporate and university campuses, disaster scenes or military operations.

\section{ADVANTAGES AND DISADVANTAGES}

Free space laser communications links eliminate the need for securing right of ways, and buried cable installations. As the equipments operate within the near infrared spectrum, they are not subject to 
government licensing and no spectrum fees have to be paid (according to Art. 7 in [3] requires only the use of the frequency spectrum below 3'000 $\mathrm{GHz}$ a licence). Additionally, since no radio interference studies are necessary, the systems are quickly deployable. The narrow laser beamwidth precludes interference with other communication systems of this type. Free space laser communications systems provide only interconnection between points that have direct line-of-sight. They can transmit through glass, however, for each glass surface the light intensity is reduced, due to a mixture of absorption and refraction, thus reducing the operational distance of a sys-tem. Occasionally, short interruptions or unavailability events lasting from some hours up to a few days can occur. Laser communication systems offer many advantages over radio frequency (RF) systems. Most of the differences between laser communication and $\mathrm{RF}$ arise from the very large difference in the wavelengths. RF wavelengths are thousands of times longer than those at optical frequencies are. This high ratio of wavelengths leads to some interesting differences in the two systems. First, the beam-width attainable with the laser communication system is narrower than that of the RF system by the same ratio at the same antenna diameters (the telescope of the laser communication system is frequently referred as an antenna).

\section{CONCLUSIONS}

The implementation of any of these systems in an inter-satellite link will require a substantial development effort. The strengths and weaknesses of the various types of lasers presently available for laser communications should be carefully considered. Based on existing laser's characteristics, the GaAlAs system, especially the full-bandwidth, direct detection system is the most attractive for inter satellite links because of its inherent simplicity ant the expected high level of technological development. The system and component technology necessary for successful inter satellite link exists today. The growing requirements for the efficient and secure communications has led to an increased interest in the operational deployment of laser cross-links for commercial and military satellite systems in both low earth and geo-synchronous orbits. With the dramatic increase in the data handling requirements for satellite communication services, laser inter satellite links offer an attractive alternative to RF with virtually unlimited potential and an unregulated spectrum.

\section{ACKNOWLEDGMENTS}

The author gratefully acknowledges for the valuable suggestion by Prof.K.R.Sontakke (Associate Professor and Dean.-Mechanical Engineering) and also by Prof. S. J. Parihar(Assistant Professor and HOD-Mechanical Engg.) and special thanks My wife Mahima A. Jaiswal for their extreme support to complete this assignment.

\section{REFERENCES}

1. IEEE communications Magazine. August 2000, free space laser communications: Laser cross-link systems and technology by: David L. Begley, Ball Aerospace \& technologies corporation

2. Free Space Optics or Laser Communication through the Air BY: Dennis Killinger Optics \& Photonics News

3. High data-rate laser transmitters for free-space laser Communications. BY:A. Biswas, H. Hemmati and J. R. Lesh Optical Communications Group Jet Propulsion Laboratory.

4. M. UllKatzmann (ed.): Laser Satellite Communications (Prentice-Hall Inc.,Englewood Cliffs, 1987).

5. H. P. Lutz: ESA Bulletin 91 (1997) 25.

6. D. L. Begley, R. A. Kobylinski, M. Ross: Intl. J. Satellite Communications 6 (1988) 91.

7. C. Chen, J. W. Alexander, H. Hemmati, S. Monacos, T. Yan, S. Lee, J. R. Lesh, S. Zingales: Proc. Free-Space Laser Communication Technologies XI, 1999 (SPIE Vol. 3615) p.142.

8. J. H. McElroy, N. McAvoy, E. H. Johnson, J. J. Degnan, F. E. Goodwin. D. M. Henderson, T. A. Nussmeier, L. S. Stokes, B. J. Peyton, T. Flattau: Proc. IEEE 65 (1977) 221.

9. G. Folcke, J. M. Defrancq, D. Geffroy: Proc. FreeSpace Laser Communication Technologies X, 1998 (SPIE Vol. 3266) p.14.

10. W. A. Atia, R. S. Bondurant: Proc. LEOS 12th Annual Meeting, San Francisco,1999, p.226.

11. V. W. S. Chan: J. Lightwave Techn. LT-5 (1987) 633.

12. M. M. Rasztovits, M. A. Schreiblehner, A. L. Scholtz, W. R. Leeb, P. Polacek: Proc. FreeSpace Laser Communication Technologies VII, 1995 (SPIE Vol. 2381) p.302. 
13. B. J. Klein and J. J. Degnan: Applied Optics 13 (1974) 2134. 11) M. Ross, P. Freedman, J. Abernathy, G. Matassov, J. Wolf, J. D. Barry: Proc. IEEE 66 (1978) 319.

14. K. Pribil, Ch. Serbe, B. Wandernoth, Ch. Rapp: Proc. Free-Space Laser Communication Technologies VII, 1995 (SPIE Vol. 2381) p.83.
15. T. Tolker-Nielsen, B. Demelenne, E. Desplats: Proc. Free-Space Laser Communication Technologies XI, 1999 (SPIE Vol. 3615) p.31.

16. K. Araki, M. Toyoda, M. Toyoshima, M. Shikatani, T. Takahashi, T. Fukazawa, Y. Arimoto: J. Communications Research Lab. 44 (1997) 209.

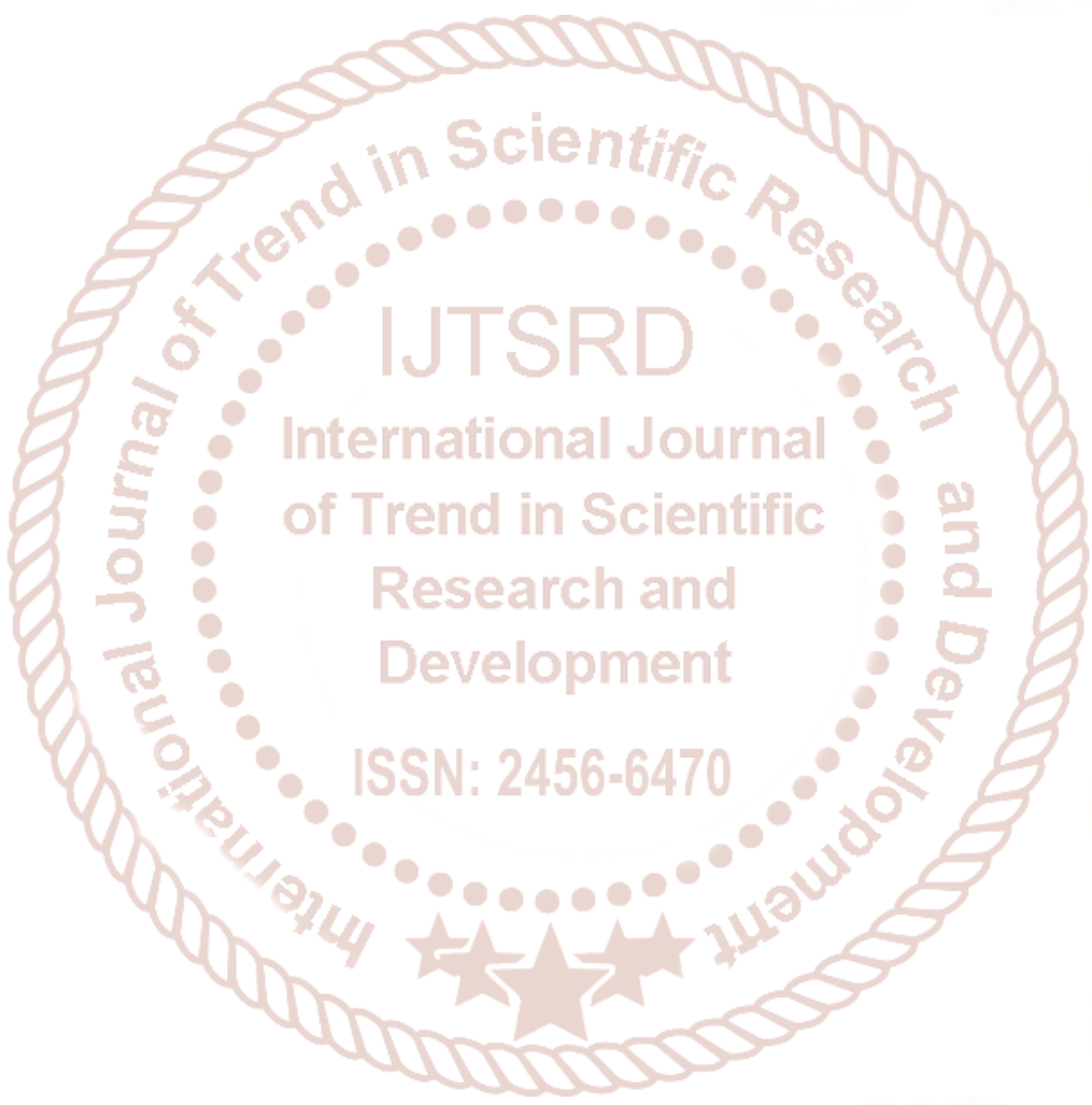

